\title{
Infección necrotizante de la pared abdominal: ¿qué hacer?
}

Necrotizing soft tissue infection on the abdominal wall. How to proceed?

\author{
Raquel M. Fresnedo-Pérez ${ }^{*}$, Ana Cabrera-Pereira ${ }^{1}$, Rubén Rodríguez-García1, Desirée Díaz-González y \\ Javier Albaladejo-Magdalena² \\ ${ }^{1}$ Servicio de Cirugía General y del Aparato Digestivo; ${ }^{2}$ Servicio de Anestesiología y Reanimación. Hospital Universitario de Cabueñes, Gijón, España
}

\begin{abstract}
Resumen
El éxito en el tratamiento de las infecciones necrotizantes de los tejidos blandos (INTB) depende de la precocidad diagnóstica y de la agresividad terapéutica, basada en el desbridamiento quirúrgico, la antibioticoterapia de amplio espectro y el soporte intensivo. Se presenta un caso de INTB secundaria a diverticulitis aguda perforada (Hinchey 4) con el fin de ilustrar las consideraciones particulares en el manejo de la INTB de localización en la pared abdominal, apoyadas en la evidencia disponible en la literatura científica. Se identifican como puntos clave la precocidad y la agresividad del desbridamiento quirúrgico, la antibioticoterapia dirigida por el cultivo y su suspensión guiada por la negativización microbiológica; y el empleo de técnicas combinadas de autoplastia y prótesis apoyadas en la terapia de presión negativa en la restauración de la pared abdominal, con lo que se ha obtenido un buen resultado.
\end{abstract}

PALABRAS CLAVE: Infección de tejidos blandos. Pared abdominal. Fascitis necrotizante. Diverticulitis aguda.

\begin{abstract}
Successful treatment of necrotizing soft tissue infections (NSTI) depends on early diagnosis and therapeutical aggressiveness, based on surgical debridement, broad spectrum antibiotics and intensive support. A case of perforated diverticulitis (Hinchey 4)-secondary-NSTI is presented in order to illustrate the particular considerations in the management of abdominal wall located NSTI, supported on the evidence available in scientific literature. Several key points are identified, such as prompt and aggressive surgical debridement; culture-guided antibiotherapy and its suspension based on microbiological negativization; and the utilization of combined techniques of autoplastic repair and replacement, also supported on negative pressure therapy in the abdominal wall restoration, which have shown a good outcome.
\end{abstract}

KEY WORDS: Soft tissue infection. Abdominal wall. Necrotizing fasciitis. Acute diverticulitis.

\section{Antecedentes}

Las infecciones necrotizantes de los tejidos blandos (INTB) constituyen un grupo de enfermedades que afectan a los tejidos cutáneos y subcutáneos, así como a las fascias superficiales y profundas y al músculo, que se caracterizan por un rápido avance de la infección, que frecuentemente evoluciona hacia una situación de sepsis o choque séptico, por lo que se requiere una actuación precoz sustentada en el desbridamiento quirúrgico agresivo, la antibioticoterapia de amplio espectro y el soporte intensivo' para evitar un desenlace fatal.

Las INTB pueden clasificarse atendiendo a diversos criterios, como la localización anatómica, la profundidad de la afectación (adipositis, fascitis, miositis) o el agente microbiano causal; o incluso a combinaciones de ambos, como agente microbiano y profundidad' ${ }^{1}$.

\section{Correspondencia:}

${ }^{*}$ Raquel M. Fresnedo-Pérez 
Según el agente microbiano, se describen tres subtipos:

- Las INTB de tipo 1 son polimicrobianas e involucran patógenos aerobios (habitualmente Streptococcus spp., Staphylococcus spp., Enterococcus spp., Enterobacteriaceae [Escherichia coli, Klebsiella spp.], Pseudomonas spp. y Acinetobacter spp.), anaerobios (Bacteroides, Clostridium spp.) $y$, en ocasiones, hongos. Se trata del subtipo más prevalente, constituyendo el $55-75 \%$ de los casos de INTB'1.

- Las INTB de tipo 2 son monomicrobianas, producidas por estreptococos del grupo A, aunque algunos autores incorporan en este grupo las infecciones causadas por Staphylococcus aureus resistente a la meticilina ${ }^{2}$.

- Las INTB de tipo 3 son monomicrobianas, debidas a Clostridium spp. o a otras especies raras y virulentas, como Vibrio vulnificus y Aeromonas spp.

La INTB de la pared abdominal puede originarse como complicación por contigüidad de un proceso intraabdominal. En estos casos, al control del foco infeccioso debe añadirse el tratamiento de la propia INTB, efectuando un control local de la infección con un adecuado desbridamiento quirúrgico y un tratamiento sistémico con soporte vital y antibioticoterapia intravenosa.

\section{Caso clínico}

Mujer de 70 años de edad, que acudió al servicio de urgencias del Hospital Universitario de Cabueñes por un cuadro de dolor abdominal, vómitos y estreñimiento de 5 días de evolución. Como antecedentes clínicos presentaba obesidad mórbida, síndrome de apnea obstructiva del sueño, hipotiroidismo yatrogénico por enfermedad de Graves-Basedow con oftalmopatía tiroidea, esteatosis hepática, enfermedad renal crónica de etiología no filiada y osteoporosis; como intervenciones previas, histerectomía con doble anexectomía, colecistectomía, tiroidectomía, trabeculectomía y artrodesis lumbar, esta última 3 semanas antes. Seguía tratamiento crónico con tiroxina $125 \mathrm{mg}$, omeprazol $20 \mathrm{mg}$, furosemida + triamtereno, prednisona $20 \mathrm{mg}$, dexketoprofeno, teriparatida, tapentadol retard 50, buprenorfina $35 \mathrm{mg}$ transdérmica y clorazepato dipotásico $5 \mathrm{mg}$.

En la exploración se objetivó una masa con signos flogóticos en la región infraumbilical, muy dolorosa a la palpación. La herida quirúrgica dorsal presentaba buen aspecto.

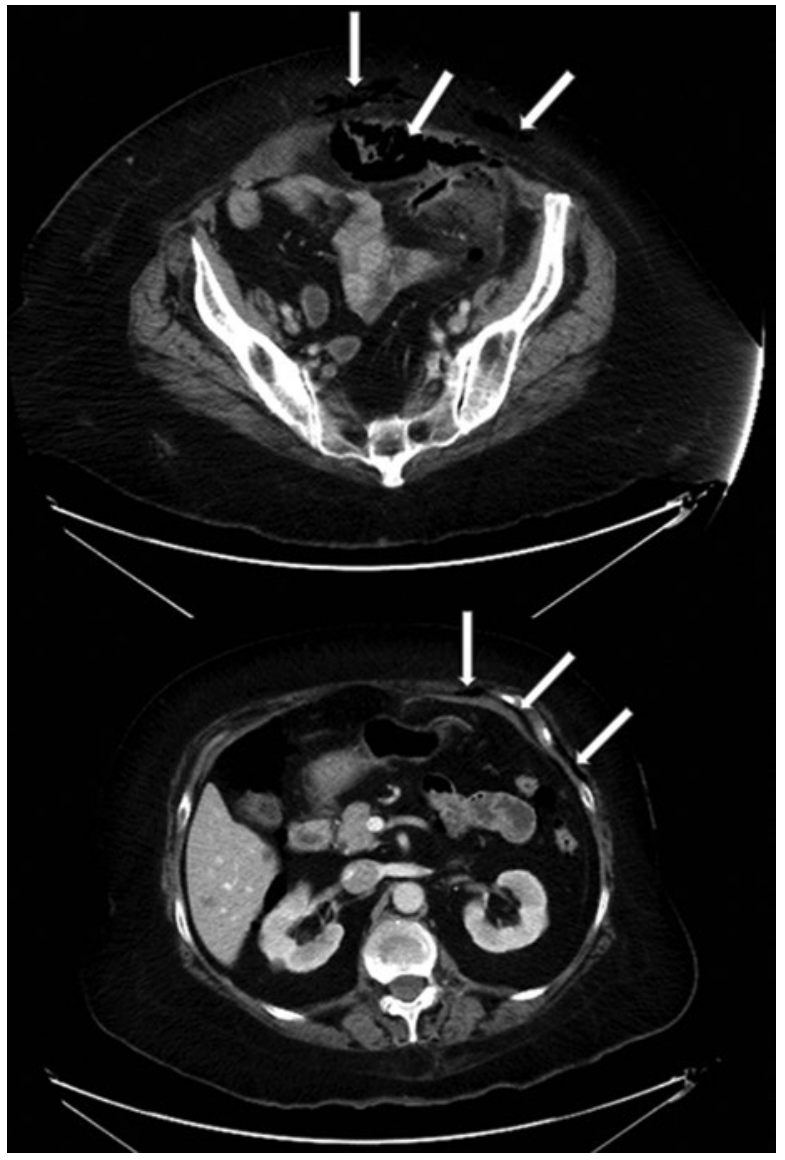

Figura 1. Cortes de tomografía computarizada en los que puede apreciarse la presencia de gas en planos subcutáneos y subfasciales.

Los resultados de laboratorio mostraron una leucocitosis leve, con neutrofilia del $92 \%$, proteína $\mathrm{C}$ reactiva (PCR) $206 \mathrm{mg} / \mathrm{l}$, lactato deshidrogenasa $607 \mathrm{U} / \mathrm{l}$, procalcitonina $3,68 \mathrm{ng} / \mathrm{ml}$, y valores de función renal habituales de la paciente. Se realizó tomografía computarizada (TC) abdominal urgente, que reveló la presencia de neumoperitoneo con pequeñas colecciones a nivel del mesosigma, engrosamiento de la fascia adyacente con múltiples divertículos y gas libre que se extendía hacia la grasa peritoneal, los planos musculares de pared abdominal anterior y el tejido subcutáneo (Fig. 1). Ante estos hallazgos ingresó en el servicio de cirugía general para ser intervenida de manera urgente por peritonitis fecal generalizada secundaria a diverticulitis sigmoidea perforada con importante infección secundaria de la pared abdominal. Se realizó sigmoidectomía-Hartmann con colostomía en el hipocondrio izquierdo y drenaje del espacio preperitoneal, del tejido celular subcutáneo y de la celda esplénica. Posteriormente fue trasladada a la unidad de reanimación, donde se registraron fibrilación auricular paroxística que requirió tratamiento con 
amiodarona, hipotensión que precisó la administración de noradrenalina, deterioro de la función renal y acidosis metabólica con hiperlactacidemia. Se comenzó tratamiento antibiótico empírico en dosis ajustadas a su función renal con imipenem y vancomicina, con mejoría clínica y analítica de la paciente.

A las 48 horas de la intervención se objetivó empeoramiento de los parámetros de sepsis en la analítica seriada (PCR 440,2 $\mathrm{mg} / \mathrm{l}$, procalcitonina $30,74 \mathrm{ng} / \mathrm{ml}$, lactato $2,2 \mathrm{U} / \mathrm{l}$ y bicarbonato $20,4 \mathrm{mEq} / \mathrm{l}$ ), y en la exploración física se constató la emisión de un exudado oscuro fétido a través de los drenajes de la pared abdominal, lo que motivó una reintervención quirúrgica inmediata. Tras la apertura de la herida quirúrgica se observó necrosis que implicaba a todos los planos de la pared abdominal, con afectación más llamativa del plano fascial, así como del tejido adiposo subcutáneo, sugestiva de fascitis necrotizante, con necrosis asociada del plano muscular (rectos anteriores y musculatura oblicua izquierda) y del propio peritoneo. Se realizó necrosectomía hasta alcanzar tejido sano, incluyendo resección cutánea, con una pérdida de sustancia de aproximadamente el $30 \%$ de la superficie de la pared abdominal anterior; se revisó asimismo la cavidad peritoneal, sin hallazgos patológicos. Se obvió el cierre de la cavidad y se efectuó una cobertura de la laparostomía con técnica de Brock, empleando una lámina de poliuretano como protección visceral, sobre la cual se dispusieron compresas empapadas en agua oxigenada, protegidas externamente por una segunda lámina de poliuretano, esta última multiperforada. La paciente fue trasladada a la unidad de cuidados intensivos.

Durante las primeras horas del posoperatorio precisó soporte hemodinámico con noradrenalina, aunque mantuvo la diuresis espontánea con cifras de insuficiencia renal reagudizada (creatinina $2 \mathrm{mg} / \mathrm{dl}$ y urea $134 \mathrm{mg} / \mathrm{dl}$ ), por lo que se sustituyó la vancomicina por linezolid para evitar nefrotoxicidad. Se realizó una nueva revisión quirúrgica en la que se objetivó ausencia de progresión de la necrosis, por lo que tras la toma de muestra para cultivo se colocó una película de polímero para protección de las asas y se fijó una malla de politetrafluoroetileno condensado de muy baja densidad $\left(\right.$ OmyraMesh $\left.{ }^{\circledast}\right)$ para evitar la retracción de la aponeurosis, con nueva cura sobre el plano fascial-malla.

A las 48 horas de la revisión se realizó otra, en la que se constató la ausencia de progresión de la infección. Se abrió longitudinalmente la malla en su parte media y se realizó una revisión de la cavidad, sin hallazgos patológicos. Se cerró la malla con aproximación de $3-4 \mathrm{~cm}$ y se repitió la cura tras la toma de muestras para cultivos.

Se programaron revisiones cada 72 horas, colocando sobre la malla un sistema de presión negativa (Vacuum Assisted Closure ABThera ${ }^{\circledR}$ ) y un catéter intraabdominal para lavados continuos con 2 litros de solución salina fisiológica cada 24 horas. En la revisión quirúrgica del décimo día se halló material fecal en el tejido subcutáneo e intrabdominal, procedente del borde de la colostomía por filtración en relación con el sistema de aspirado, por lo que se decidió no recolocarlo nuevamente. Se realizó un lavado exhaustivo de la cavidad y se colocó un sistema de lavado, retirando la malla previa y colocando una nueva malla de polipropileno y poliglecaprona (PhysioMesh ${ }^{\circledR}$ ).

Los primeros cultivos reportaron la presencia de Pseudomonas aeruginosa, Fusobacterium varium, E. coli y Enterococcus faecalis, cubiertos con la pauta de imipenem + vancomicina/linezolid. Posteriormente se detectaron Candida albicans y Clostridium perfringens, por lo que se adicionaron anidulafungina y clindamicina al tratamiento. Cada antibiótico fue suspendido tras la negativización del agente infeccioso al cual iba dirigido en los cultivos seriados.

A los 32 días de la necrosectomía se obtuvieron cultivos estériles y se realizó TC de control que informó de la ausencia de hallazgos patológicos. Los resultados de laboratorio mostraron normalización de los parámetros de sepsis y restauración de la función renal basal de la paciente, que fue dada de alta de la unidad de cuidados intensivos y pasó a planta de hospitalización de cirugía general.

Durante su estancia en planta se colocó nuevamente el sistema de presión negativa, con desarrollo de tejido de granulación, aunque con dehiscencia del plano fascial. Se planteó el cierre mediante colgajos musculocutáneos, pero la técnica fue desestimada por el servicio de cirugía plástica dados los antecedentes médicos de la paciente, por lo que se mantuvo el sistema de presión negativa hasta el cierre cutáneo, a las 18 semanas de la necrosectomía. Durante este periodo la paciente recibió tratamiento rehabilitador y suplementación nutricional, y fue dada alta a su domicilio 2 semanas después del cierre cutáneo, en buen estado general, tras completar un total de 20 semanas de ingreso hospitalario.

\section{Discusión}

En la literatura disponible en torno a la INTB se incide específicamente en la necesidad de un 
diagnóstico precoz, considerando que la clave de un tratamiento exitoso radica en la rápida toma de decisiones. Disponemos de herramientas para apoyar el diagnóstico de INTB, como el Laboratory Risk Indicator for Necrotizing Fascitis Score (LRINEC), basado en los valores de PCR, recuento leucocitario, hemoglobina, sodio, creatinina y glucosa, que atribuye un valor predictivo positivo del $92 \%$ y un valor predictivo negativo del $96 \%$ de INTB si se determina una puntuación de 6 o más puntos. Sin embargo, aunque existen diversos estudios con el objetivo de validar esta herramienta para el diagnóstico, sus resultados sugieren que su utilidad es mayor en la predicción pronóstica'. Por otra parte, las pruebas de imagen tienen una utilidad limitada, ya que la TC solo es válida cuando puede objetivarse gas en el tejido, y la resonancia magnética, si bien permite diferenciar entre infecciones necrotizantes y no necrotizantes, supone un consumo de tiempo que no es asumible en estos casos. Así pues, el diagnóstico debe ser eminentemente clínico y quirúrgico ${ }^{3}$.

En el caso reportado es posible identificar entre los antecedentes clínicos factores predisponentes al desarrollo de INTB, como son la obesidad mórbida, el hipotiroidismo, el fallo renal crónico, el estrés quirúrgico reciente y el tratamiento con esteroides, además de la edad superior a 60 años ${ }^{1}$, lo cual, unido a los hallazgos quirúrgicos (y a un LRINEC de 9), es suficiente para considerar el diagnóstico de INTB y justifica el desbridamiento quirúrgico precoz y agresivo como primera maniobra terapéutica, teniendo en cuenta que la prontitud y la agresividad de la necrosectomía constituyen los factores más determinantes de la morbimortalidad esperable, que se sitúa entre un 30 y un $70 \%$ de los casos, estando el incremento en relación directa con la demora quirúrgica ${ }^{4}$, así como con la edad del paciente y la presencia de dos o más comorbilidades ${ }^{5}$. Por otra parte, la presencia en la TC de gas en los espacios preperitoneal, prefascial y retrofascial, y subcutáneo, apoya el diagnóstico y hace innecesario realizar una nueva prueba de confirmación ante los hallazgos clínicos a las 48 horas de la intervención, lo cual supondría una demora quirúrgica inasumible. Por otra parte, dicho hallazgo plantea la cuestión de que posiblemente hubiese estado indicado un desbridamiento más amplio en la primera intervención, con resección del tejido afecto, en lugar de un drenaje simple. En la revisión multicéntrica de Faraklas et al. ${ }^{3}$, de 2017, se incide en la agresividad de la necrosectomía, que debe extenderse hasta hallar tejido sangrante en cualquier circunstancia, y que bajo ningún concepto debe cerrarse. La revisión se indica a las 24 horas en todas las situaciones para comprobar si existe progresión de la necrosis, o antes si las condiciones clínicas del paciente así lo precisan. En este sentido, la localización en la pared abdominal de la INTB no debe modificar el enfoque terapéutico, incluyendo en la resección los tejidos borderline, aunque suponga una pérdida de sustancia mutilante que deberá ser restaurada en un tiempo posterior ${ }^{4}$.

Existe evidencia clara de que el tratamiento de primera línea de la INTB está constituido por el desbridamiento quirúrgico y el tratamiento antibiótico de amplio espectro ${ }^{3}$. Sin embargo, no disponemos de recomendaciones sólidas en torno al tiempo que debe mantenerse la antibioticoterapia. La mencionada revisión de Faraklas, et al. ${ }^{3}$ señala que no existe evidencia de que un tratamiento antibiótico prolongado condicione mejores resultados, siendo el factor determinante el control del foco séptico. En este sentido, hemos estimado que la manera más adecuada de valorar el control de foco es realizar cultivos seriados, tomando muestras en cada una de las revisiones quirúrgicas. Esto posibilita, por una parte, modificar el tratamiento antibiótico para dirigirlo a los patógenos aislados, y por otra parte, proceder a la suspensión de cada fármaco concreto cuando se haya constatado la negativización del patógeno al cual iba dirigido. Con esta estrategia se consigue limitar el uso de antibióticos de amplio espectro y minimizar los efectos adversos de su uso prolongado. En este sentido, consideramos una opción interesante sustituir la vancomicina, que habitualmente se propone como tratamiento para microorganismos grampositivos ${ }^{1}$, por el linezolid, de espectro similar, en los pacientes con enfermedad renal previa o con afectación renal durante el proceso, para evitar el riesgo de nefrotoxicidad de la vancomicina.

Una vez establecido el control del foco, y conseguida la estabilización del paciente, consideramos que la obtención de cultivos estériles puede ser un buen indicador del momento de plantear la restauración de la pared abdominal. En nuestro caso empleamos una malla con la doble función de actuar como interfaz entre el sistema de presión negativa y las asas, y de evitar la retracción de los bordes aponeuróticos, posibilitando una aproximación progresiva de estos. La contaminación fecal por aspiración desde el borde de la colostomía limitó el uso de la terapia de presión negativa que, de otro modo, como está constatado en la literatura, constituye un planteamiento eficaz en el 
abdomen abierto ${ }^{6,7}$. Por otra parte, las técnicas de cierre mediante colgajos e injertos requieren una valoración cuidadosa e individualizada por parte del servicio de cirugía plástica, teniendo en cuenta los aspectos propios del defecto, así como las condiciones del paciente y sus antecedentes medicoquirúrgicos, lo cual limita su aplicación.

\section{Conclusión}

El diagnóstico precoz, que debe ser fundamentalmente clínico y quirúrgico, y una rápida toma de decisiones son claves para el éxito del tratamiento de las INTB de la pared abdominal. Los pilares de dicho tratamiento son el desbridamiento quirúrgico agresivo, la antibioticoterapia de amplio espectro y el soporte intensivo del paciente, debiendo realizar revisiones quirúrgicas seriadas para verificar la ausencia de progresión de la necrosis. Una vez se disponga de los resultados de los cultivos, la antibioticoterapia puede dirigirse a cada agente infeccioso de una manera más específica, ajustándose para evitar, en medida de lo posible, sus potenciales efectos adversos.

Solo cuando se ha establecido el control del foco infeccioso y el paciente se encuentra estable puede plantearse la reconstrucción de la pared abdominal, que debe abordarse de forma individualizada en cada caso, teniendo en cuenta la pérdida de sustancia, el estado del paciente y sus antecedentes clínicos.
La observación rigurosa de las recomendaciones en estos casos puede evitar un desenlace fatal, incluso en casos de mal pronóstico como el reportado.

\section{Conflicto de intereses}

No existen conflictos de interés de los autores.

\section{Financiación}

No se ha recibido financiación para llevar a cabo este trabajo.

\section{Bibliografía}

1. Cocanour CS, Chang $P$, Huston JM, Adams CA, Díaz JJ, Wessel CB, et al. Management and novel adjuncts of necrotizing soft tissue infections. Surg Infect. 2017;18:250-72.

2. Sarani B, Strong M, Pascual J, Schwab CW. Necrotizing fasciitis: current concepts and review of the literature. J Am Coll Surg. 2009;208:279-88.

3. Faraklas I, Yang D, Eggerstedt M, Zhai Y, Liebel P, Graves G, et al. A multi-center review of care patterns and outcomes in necrotizing soft tissue infections. Surg Infect. 2016;17:773-8.

4. Marinis A, Voultsos M, Foteinos A, Tselioti $P$, Avraamidou A, Paschalidis N, et al. Necrotizing soft tissue infection of the right anterolateral abdominal wall caused by a ruptured gangrenous appendix in an elderly diabetic patient. Infez Med. 2015;23:182-6.

5. Smith NE, McKenney M. Acute sigmoid diverticulitis presenting as necrotizing fasciitis of the thigh and retroperitoneum. Am Surg. 2016; 82:301-2.

6. Tolonen $M$, Mentula $P$, Sallinen V, Rasilainen S, Bäcklund $M$, Leppäniemi A. Open abdomen with vacuum-assisted wound closure and mesh-mediated fascial traction in patients with complicated diffuse secondary peritonitis: a single-center 8-year experience. J Trauma Acute Care Surg. 2017;82:1100-5.

7. Acosta S, Bjarnason T, Petersson U, Pålsson B, Wanhainen A, Svensson M, et al. Multicentre prospective study of fascial closure rate after open abdomen with vacuum and mesh-mediated fascial traction. $\mathrm{Br}$ J Surg. 2011;98:735-43. 